\title{
Aplicación del enfoque de problemas a la diabetes mellitus de tipo 2 en educación médica: una revisión integrativa
}

\author{
Juan M. Muñoz Cano, Crystell Guzmán Priego y Juan A. Córdova Hernández \\ División Académica Ciencias de la Salud, Universidad Juárez Autónoma de Tabasco, Villahermosa, Tabasco, México. \\ (correo-e: juan.munoz@ujat.mx; crystell.guzman@ujat.mx; juan.cordova@ujat.mx)
}

Recibido Nov. 15, 2019; Aceptado Ene. 16, 2020; Versión final Feb. 20, 2020, Publicado Jun. 2020

\begin{abstract}
Resumen
Analizar la literatura científica de diabetes mellitus de tipo 2 para caracterizar la causa raíz principal y las mejores estrategias de solución y prevención en los procesos educativos. El análisis se hizo mediante el Método para la Solución de Problemas en Ocho Etapas. Para ello se preparó un guion a partir de la definición de los conceptos de interés. Se definieron los criterios de búsqueda de la literatura. Posteriormente se seleccionó la información con esos criterios. Se identificaron las medidas de contención y los determinantes sociales que los minimizan y se identificaron las principales soluciones de aplicación en la práctica de primer nivel de atención. También se evaluó la pertinencia de modificaciones en los procesos educativos con base en acotar la causa raíz principal de las enfermedades no transmisibles, la adición de fructosa a bebidas y alimentos de la industria. Se encontró que diabetes y obesidad no son un simple desequilibrio de calorías. La salud depende de la comida étnica tradicional. Estos conceptos deben incorporarse a los procesos de formación de profesionales de la salud.
\end{abstract}

\section{Application of the problem approach to diabetes mellitus type 2 in medical training: an integrative review}

\begin{abstract}
The purpose of this study was to perform a literature review about diabetes mellitus 2 to characterize the main root cause and the best strategies for solution and prevention in the educational processes. The analysis was performed using the Eight Disciplines Problem Solving Method. To this end, a script was prepared based on the definition of the concepts of interest. The search criteria for data were also defined. The information was subsequently selected using these criteria. This study identified the containment measures and the social determinants that minimize them and the main solutions for practical application at the primary care level. The relevance of changes in educational processes based on the cause main root were examined for noncommunicable diseases, addition of fructose to soft drinks, and foods made by the food industry. The results showed that diabetes and obesity are not the result of simple calorie imbalance. Good health depends on traditional ethnic dishes. These concepts should be incorporated into the training processes of health professionals.
\end{abstract}

Keywords: prevention; root cause; fructose; medical education; patient care 


\section{INTRODUCCIÓN}

La diabetes mellitus de tipo 2 (DM2) es una enfermedad caracterizada por hiperglucemia que ha tenido recientes incrementos dramáticos en su prevalencia. La aparición de pacientes ha aumentado en grupos de adultos jóvenes, adolescentes y niños lo cual sobrepasa las proyecciones (King et al, 1988). El incremento de la prevalencia de DM2 y otras enfermedades no transmisibles (ENT) es causa de cuatro de cinco muertes en las Américas (Pan American Health Organization PAHO, 2017). Para explicar la pandemia de DM2 se menciona la obesidad como primer factor de riesgo. El incremento en personas con obesidad se explica por el desbalance entre las calorías que se consumen y las que se gastan en actividad física, por lo cual se asume que el sedentarismo es una causa principal. Otra causa en la explicación dominante es el aumento de la esperanza de vida, que en las Américas ha aumentado 16 años en los últimos 45 años, 88\% de la población vive más de 60 años y de esos, 42\%, más de 80 años (PAHO, 2017).

Este razonamiento tampoco considera que una alta proporción de pacientes con DM2 no presenta obesidad y que la resistencia a la insulina y la obesidad central probablemente sean problemas concurrentes con una causa común (Kahn y Flier, 2000). Por otra parte, el rápido incremento no se explica a causa de posibles modificaciones en la carga genética porque ha ocurrido en un periodo demasiado pequeño de tiempo para sustentar esta causa. Esto también sucede con enfermedad isquémica del corazón, enfermedad vascular cerebral, hipertensión, cirrosis hepática no alcohólica no autoinmune, enfermedad renal crónica, y varios tipos de cáncer asociados a la dieta moderna (Zimmet et al, 2016).

Estos argumentos no pueden explicar el incremento $\mathrm{n}$ la prevalencia de estas enfermedades. DM2 como otras ENT tiene inicio subclínico (pero detectable mediante pruebas de laboratorio) decenios antes del diagnóstico clínico. Por ejemplo, la historia natural de la DM2 inicia al menos 20 años antes de la observación de hiperglucemia. Las anomalías más tempranas que se pueden detectar de manera rutinaria en ese momento son, por ejemplo, modificaciones en la respuesta a la insulina. La resistencia a la hormona se presenta ya que, aunque el páncreas tiene capacidad de liberar insulina, se requieren cada vez mayores cantidades de la misma para evitar la hiperglucemia. El diagnóstico de resistencia a la insulina, como en el HOMA-IR, es el resultado de glucosa en ayunas por insulina en ayunas entre una constante. Por otra parte, las enfermedades no transmisibles tienen como fondo la inflamación de bajo grado. Esto puede detectarse años antes de que se presente la hiperglucemia mediante la medición de proteína C reactiva, II-6, NFk $\beta$, entre otros marcadores. Éstos se asocian a un estilo de vida donde predomina la comida industrializada moderna y se han abandonado los patrones de consumo de alimento tradicional (Tabung et al. 2016), y que predicen anomalías metabólicas y ENT (Ruiz et al, 2017).

\section{Procesos educativos y la pandemia de DM2 y ENT}

El cambio de estilo de vida es fundamental para prevenir o retrasar la aparición de la DM2 en pacientes en riesgo. El manejo no farmacológico, que incluye mayor consumo de alimentos de tipo tradicional pues tienen menor índice glucémico y mayor aporte de fibra y actividad física para mejorar la sensibilidad a la insulina, son consideradas en las Guías Clínicas como piedra angular del manejo de los pacientes con DM2 y otras ENT pues mejoran los valores de tensión arterial y disminuye el riesgo cardiovascular. Desafortunadamente esto no se realiza de manera rutinaria, lo que se expresa como progresión de la mala filtración glomerular, la disminución del flujo sanguíneo a causa de aterosclerosis, y comorbilidades como hígado graso y cirrosis, hipertensión arterial, varios tipos de cáncer, declinación cognitiva, que se encuentran como parte de la transición epidemiológica donde la mayor parte de las causas de muerte y de pérdida de años saludables corresponde a ENT que pueden ser prevenidas. Por ello el objetivo de esta investigación es analizar la literatura acerca de las estrategias de contención al incremento de la prevalencia de DM2, identificar una causa raíz principal y estrategias de solución y prevención que fortalezcan los procesos de formación en salud.

\section{METODOLOGÍA}

Se hizo una revisión integrativa (Whittemore y Knafl, 2005). El estudio se hizo mediante la aplicación del Método para la Solución de Problemas en Ocho Etapas, 8D. El método permite identificar una causa raíz principal y soluciones que van más allá de las capacidades de una disciplina (Kaplik et al., 2013). La selección de la literatura se hizo en cuatro fases: 1) Preparación de un guion a partir de la definición de los conceptos incluidos en los pasos del modelo 8D. 2) Definición de los criterios de búsqueda de la literatura. Los artículos se seleccionaron de acuerdo con el tipo de participantes de los estudios, el tipo de intervención y los resultados de interés. 3) Colecta de datos con énfasis en los artículos con enfoque cuantitativo y diseño experimental o quasi experimental. También los estudios de observación y análisis de poblaciones. 4) Análisis crítico de los estudios incluidos para cada concepto identificado en el guión. 
Los criterios de exclusión fueron: publicaciones duplicadas, estudios de caso o sólo casos publicados. Otro criterio de exclusión fue que los autores tuvieran conflictos de interés. El conflicto se refiere a recibir tesis para estudios de doctorado, aportaciones para las fundaciones de los autores acerca de diabetes o nutrición, ser consultores asalariados de la industria del azúcar, entre otras percepciones económicas (Rey y González, 2018; Mozzafarian, 2017). La búsqueda se realizó en abril de 2018. Una vez identificada la raíz principal se hizo una búsqueda en PUBMED con los términos ("fructose"[MeSH Terms] OR "fructose"[All Fields]) AND ("diabetes mellitus"[MeSH Terms] OR ("diabetes"[All Fields] AND "mellitus"[All Fields]) OR "diabetes mellitus"[All Fields]) AND ("review"[Publication Type] OR "review literature as topic"[MeSH Terms] OR "review"[All Fields]). La búsqueda obtuvo 246 items donde se localizaron 43 que se referían de manera específica a los efectos metabólicos de la fructosa en la historia natural de la DM2. De ellos se eliminaron cinco por el criterio de exclusión de financiamiento por la industria. Con los datos de estas 38 revisiones se construyó el apartado "causa raíz principal".

\section{RESULTADOS}

Las proyecciones hechas a finales del siglo XX estimaban la prevalencia de $5.5 \%$ de la población mundial con diabetes para 2025 (King et al., 1988). Sin embargo, la prevalencia global para 2014 fue de 8.5\% (WHO, 2016). Esta tendencia se confirma en distintos estudios realizados. Por ejemplo, en 2017 se estimó en 86 millones las personas con prediabetes sólo en Estados Unidos, más de uno de cada tres habitantes adultos de ese país (American Diabetes Association [ADA], 2019). Las modificaciones en el perfil de mortalidad como de la carga de la enfermedad muestran un rápido aumento de las que se agrupan dentro de la categoría de ENT. Para el caso de la DM2 la carga de estas enfermedades muestra un aumento en los años de vida ajustados por discapacidad (AVAD). En México, DM2 tuvo AVAD en hombres de 280,985 en 1990. Ésta aumentó $98 \%$ en 2010, ya que se calcularon 557,925 años. Para las mujeres AVAD de DM2 fue de 324,988 en 1990 y 526,724 en 2010 con aumento del 57\% (Lozano et al., 2013).

\section{Descripción de las acciones de contención}

Las acciones orientadas a contener el incremento de la prevalencia de T2DM son la aplicación de regímenes alimenticios, prescripción de actividad física, medicamentos, etiquetado a alimentos y bebidas, restricción de venta en espacios educativos y de salud, impuestos a bebidas con azúcar o edulcorantes artificiales (Figura 1). En conjunto estas medidas no han sido efectivas para disminuir la prevalencia de obesidad clase 3 (Flegal et al., 2016), ni de DM2 y de otras ENT (King et al, 1998). En los menús que habitualmente proporciona el personal de nutrición se detallan tres comidas y dos colaciones, el conteo de calorías y la equivalencia de alimentos. Éstas no son comprendidas por la población en general. En México, la 2016 la Encuesta Nacional de Salud y Nutrición de Medio Camino (ENSNMC) mostró que 73.6\% de la población desconocía la cantidad de calorías que debería consumir, por consiguiente, desconocía el manejo de este tipo de prescripción (Instituto Nacional de Salud Pública [INSP], 2016). La recomendación no considera la liberación de insulina donde unos alimentos producen un pico muy alto en la primera fase (por absorción rápida) y otros una liberación sostenida en la segunda (por absorción lenta) (Rorsman et al., 2000). La equivalencia de alimentos de MyPlate de Estados Unidos no consideró que la mayor proporción de calorías debe tener base en cereales integrales: "debería haber un claro mensaje de que la población disminuya el consume de carnes rojas, queso, mantequilla, y azúcar, pero eso ofendería a la industria ponderosa” (Willet y Ludwig, 2011). Estos regímenes dietéticos tienen como resultado lo contrario de su propósito y los pacientes ganan peso a largo plazo (Mozaffarian, 2016).

La recomendación de actividad física para disminuir la masa corporal y afrontar T2DM no toma en cuenta las calorías que se consumen por minuto por cada tipo de actividad física. Una persona de $70 \mathrm{~kg}$ consume durante media hora 367.5 kilocalorías al trotar a velocidad de 9 a $10 \mathrm{~km} / \mathrm{h}, 294 \mathrm{kcal}$ con baile aeróbico, $294 \mathrm{kcal}$ al nadar a $4 \mathrm{~km} / \mathrm{h}$ (Abellán et al., 2014, p. 64). Estas no son capacidades que la mayoría de la gente tiene, o las que se pueden obtener a menos de establecer un programa de entrenamiento de mediano rendimiento.

Se han prescrito diversos fármacos como orlistat para disminuir la masa corporal sin que existan estudios que muestren consistentemente los efectos benéficos. El único efectivo es metformina, fármaco para DM2, ha sido prescrito para disminuir la masa corporal sobre todo en pacientes con índice de masa corporal (IMC) $\geq 35$ $\mathrm{kg} / \mathrm{m}^{2}$ para disminuir la probabilidad de desarrollar DM2 (Aroda et al., 2017). La metformida se asocia a muchos efectos biológicos benéficos, incluso antienvejecimiento (Barzilai et al., 2016).

En México se puso en marcha en 2014 un impuesto a bebidas dulces. Ha tenido buenos resultados en disuadir a los consumidores y se ha propuesto su puesta en marcha en otros países, como Chile, Reino Unido y Australia (Cobiac et al., 2017). Es posible que en algún momento se implante el etiquetado monocromático para los productos ricos en azúcar. Mientras tanto en algunos países se ha tenido éxito en la implantación de un etiquetado con base en colores que identifican la carga calórica del producto. Este etiquetado varía entre 
un color para el alimento con base en los ingredientes totales o un color para cada ingrediente. Ecuador que implantó el etiquetado en 2014, tiene leyendas del tipo "bajo en sal, no contiene azúcar, no contiene grasa" para el color verde. En México el etiquetado muestra un porcentaje de las calorías de azúcares agregados, grasas y sal, como una meta y no una limitación. Este etiquetado fue "comprensible" para 13.8\%, "algo comprensible" para $30.5 \%$ y "poco a nada" para $55 \%$ de la población que participó en la ENSNMC de México (INSP, 2016). En la misma encuesta la frecuencia con que los consumidores compraron productos con base en la información de los empaques fue sólo $6.7 \%$ para "casi siempre" y 5.4\% para "siempre". Esta es también la limitante para el etiquetado de Reino Unido que se implantó en 2012 que, si bien muestra los colores del semáforo, se colocan con base en el contenido de azúcar añadida, sal y grasas saturadas como porcentaje de requerimientos, lo cual no debe ser el objetivo primordial del etiquetado.

Tabla 1. Comparación de la información y las acciones

\begin{tabular}{|c|c|c|}
\hline $\begin{array}{l}\text { Categorías } \\
\text { análisis }\end{array}$ & $\begin{array}{l}\text { Emanada de datos científicos y políticas } \\
\text { públicas }\end{array}$ & $\begin{array}{l}\text { Producida por la industria y científicos con } \\
\text { conflicto de interés }\end{array}$ \\
\hline $\begin{array}{l}\text { Medidas de } \\
\text { contención }\end{array}$ & $\begin{array}{l}\text { Aumento de impuestos a bebidas y } \\
\text { alimentos calóricos. Mejores etiquetados } \\
\text { para la identificación productos con } \\
\text { niveles altos de calorías, fructosa, sodio. } \\
\text { Restricción del expendio de productos en } \\
\text { espacios educativos y en edificios del } \\
\text { sector salud. }\end{array}$ & $\begin{array}{l}\text { Expendios de productos de la industria en las } \\
\text { rutas de los estudiantes a las escuelas. Falta de } \\
\text { cumplimiento en las propuestas de } \\
\text { autorregulación por parte de la industria. }\end{array}$ \\
\hline Causa raíz principal & $\begin{array}{l}\text { Datos acerca de los efectos de la fructosa } \\
\text { en los procesos metabólicos se generaron } \\
\text { en diversos estudios desde los años } 60 \text { s. } \\
\text { En esa época la industria del azúcar } \\
\text { desvió los objetivos de las investigaciones } \\
\text { hacia los lípidos lo que ralentizó la } \\
\text { aparición de nuevos datos. }\end{array}$ & $\begin{array}{l}\text { Hay múltiples datos aportados por investigadores } \\
\text { con conflictos de interés en forma de } \\
\text { subvenciones, becas para estudios de posgrado, } \\
\text { puestos en consejos de administración. }\end{array}$ \\
\hline $\begin{array}{l}\text { Implementación de la } \\
\text { solución }\end{array}$ & $\begin{array}{l}\text { Transitar paulatinamente a patrones de } \\
\text { consumo de bebidas y alimentos con de } \\
\text { las cocinas étnicas tradicionales, } \\
\text { productos locales y con menores } \\
\text { impactos sobre el ambiente. }\end{array}$ & $\begin{array}{l}\text { En sus publicaciones afirman que los estudios no } \\
\text { han podido demostrar una dosis de fructosa que } \\
\text { sea peligrosa, ya que en sus metaanálisis no se } \\
\text { encuentran estudios homogéneos y de alta } \\
\text { calidad. }\end{array}$ \\
\hline
\end{tabular}

En octubre de 2014 los ministros de salud de toda la región de las Américas se comprometieron a restringir la venta de alimentos procesados y aumentar la de alimentos saludables. Para esto son necesarias medidas en concordancia con la industria (Popkin y Kenan, 2016). Sin embargo, el posible efecto beneficioso de estas medidas queda neutralizado por la posibilidad de consumir alimentos y bebidas calóricas en las cercanías de las escuelas (Cutumisu et al., 2017; Caraher et al., 2016; Missbach et al., 2016). También porque muchas de esas medidas como las de autoregulación no modifican sus sistemas de producción ni sustancialmente sus medios de promoción y marketing (Henderson et al., 2017).

\section{Análisis, identificación y verificación de la causa raíz}

Las estimaciones de finales del siglo XX esperaban 5.5\% de la población mundial con diabetes para 2025. Los nuevos casos se presentarían a sobre todo a partir de los 45 años con la mayor parte de la prevalencia entre 45 y 65 años (King et al., 1998). Pero estas estimaciones pronto fueron obsoletas ante lo dramático del incremento de los casos nuevos. En México donde la población de personas mayores de 45 años no fue mayor al $20 \%$ en 2016 , el envejecimiento no explica por qué la prevalencia fue $9.4 \%$ para la población mayor de 20 años (NPHI, 2016). La DM2 tiene inicio muchos años antes del diagnóstico, hasta 25 años antes de cambios metabólicos previos al inicio de la resistencia periférica a la insulina, entre ellos la inflamación de bajo grado (Spranger et al., 2003). La identificación de la DM2 en edades avanzadas significa el retraso en el diagnóstico a partir de esas anomalías tempranas (DeFronzo, 2009).

El sedentarismo se asume como otro factor primordial. En México en 1999 la obesidad en niños de 5 a 11 años era 18.6\%, aumentó a 26\% en 2006 y a 33.2 en 2016 (NPHI, 2016). A esta edad normalmente los niños tienen actividades que no son deportivas, son muy activos, y se ha documentado el efecto de las costumbres de las familias en el desarrollo de la obesidad antes de que los niños ingresen a la escuela elemental. Para la etapa escolar sólo $17.2 \%$ de los niños en México realizan actividad física vigorosa 60 minutos los siete días de la semana (INSP, 2016). Aunque es significativo el bajo porcentaje de niños activos, algunos estudios no encuentran correlación entre sedentarismo y obesidad infantil (Edo et al., 2010). Para el grupo de 
adolescentes en México, 60.9\% cumplieron en 2016 con al menos 420 minutos de actividad física de moderada a vigorosa, sin embargo, la proporción de sobrepeso-obesidad combinada en este grupo fue de $36.3 \%$ sin que se haya reportado la proporción de adolescentes con sobrepeso-obesidad que hacían actividad física de moderada a vigorosa (INSP, 2016). Esto podría suceder donde más que la inactividad como causa de obesidad es la obesidad la que se relaciona con inactividad lo cual explica por qué fracasan las intervenciones encaminadas a generar actividad física en niños con el propósito de hacerlos disminuir el IMC (Metcalf et al., 2011). Esto es una barrera para la población en general pues en México $27.5 \%$ de quienes respondieron a la MNHNS dijeron que no hacen actividades físicas debido a problemas de salud (INSP, 2016).

\section{La causa principal}

Esta parece ser el alto contenido de fructosa en la dieta moderna (Figura 1). Los efectos del mayor consumo de azúcar como base de la pandemia de ENT se reportó desde los 60s. Posteriormente se hicieron observaciones acerca de que el consumo de azúcar y no de grasa se correlacionaba más con el incremento de la prevalencia de obesidad asociada a ENT, aunque hubo manipulaciones a la información científica, inducidas por la industria, para que fuera la grasa y no el azúcar lo que se proscribiera de las dietas recomendadas como saludables (Kearns et al, 2016). Las perturbaciones en los patrones de consumo de alimentos, con más azúcar visible u oculta, generaron la migración de las enfermedades de los estratos de mayores ingresos a los pobres, quienes ahora están sujetos a las enfermedades infecciosas y también a las ENT (Peña y Bacallao, 2000). Los efectos de la fructosa se encuentran varios niveles. En hipotálamo donde modifica la percepción del "gusto" por los alimentos lo que podría explicar la preferencia por bebidas endulzadas con azúcar sobre edulcorantes artificiales (Delogu et al., 2016). En el hígado donde produce cambios que conducen a la cirrosis hepática no alcohólica y se produce más ácido úrico a partir del AMP.

El azúcar se agrega a alimentos, sobre todo del tipo de repostería industrial, como a bebidas. En México, 21\% del consumo total de energía de los adolescentes proviene de bebidas endulzadas (Dehghan et al., 2008; Rivera et al., 2008). En este país, aunque $93 \%$ de la gente considera que los refrescos no son saludables y se asocian a diabetes, son agradables para el 83\% (INSP, 2016). El consumo de azúcar es adictivo y ese efecto se induce desde la etapa intrauterina (Goran et al., 2013). Su efecto primordial está sobre centros neurales superiores donde crea cambios en la percepción y se transita de una alimentación por necesidades biológicas a otra donde el alimento dulce es una recompensa. El aumento de azúcares produce aumento del número y tamaño de los adipocitos. Esto tiene consecuencias en la percepción de la saciedad. En individuos sanos una vez que se come se libera leptina en el tejido adiposo. Su efecto está sobre limitar la ingestión de alimento. Pero si aumenta la masa de tejido adiposo, se libera más leptina que satura los receptores y produce falla en la respuesta (Teff et al., 2004).

En Estados Unidos hay prevalencia de enfermedad de hígado graso no alcohólico (NAFLD por sus siglas en inglés), de 20 a 30\% en la población, de 80 a 100 millones de personas (Loomba y Sanyal, 2013). Este es el antecedente más común de enfermedad crónica hepática (Sayiner et al., 2016). Para el papel de la fructosa en la NAFLD hay un modelo de dos hits (Lim et al., 2010). El primero es su alto consumo, pues se deposita como ácidos grasos en hígado (Lim et al., 2010; Basaranoglu et al., 2013; Nomura et al., 2012). El segundo hit en la progresión de la enfermedad hepática es el estrés oxidativo asociado a peroxidación de ácidos grasos, pues aumentan especies reactivas de oxígeno; también la activación de las citosinas, el óxido nítrico y las toxinas endógenas o metabolitos de la fructosa pues una consecuencia es la fructosilación de proteínas (Nomura et al., 2012).

La ingesta excesiva de fructosa, mayor a $50 \mathrm{~g}$ al día, se asocia a una mayor síntesis de ácido úrico (Johnson et al., 2009; Johnson et al., 2013; Rodrigo et al., 2016). Una vez en el sistema, la fructosa entra a las células por transportadores de membrana como Glut 5 o Glut 2. Allí es metabolizada por fructocinasa, la que no tiene un medio de sistema de represión por retroalimentación. Esto produce depleción del sustrato para fosforilación con lo que el ATP se metaboliza a AMP. Ello produce activación de la deaminasa de AMP, y generación de ácido úrico el cual inhibe el NO endotelial, así como la producción de anti-oxidantes inducidos por ácido úrico, producción de proteína-C-reactiva e inflación crónica de bajo grado. De esta manera, la fructosa actúa por la vía clásica de incremento de ingreso calórico como por vías metabólicas diferentes (Johnson et al., 2009). Se sabe que el ácido úrico es un factor fuertemente asociado a DM2 y síndrome metabólico desde la etapa prenatal (Johnson et al., 2009; Rodrigo et al., 2016). En pacientes con DM2 es un fuerte predictor de enfermedad cerebrovascular (Lehto et al., 1998) e hipertensión (Nakanishi et al., 2003).

En otro aspecto, la modificación de las dietas tradicionales como la Mediterránea y su sustitución por la de tipo moderno, produce importantes perturbaciones en la microbiota intestinal por cambios en el aporte de prebióticos con la consecuencia de liberación de compuestos tóxicos desde el colon y la pérdida de la producción de moléculas protectoras para el colon como propionato, butirato y acetato por la microbiota, lo cual es causa de NAFLD y cáncer de colon, entre otras patologías (Panasevich et al., 2017). Uno de los 
efectos estudiados es la diminución de Bifidobacteria, Lactobacillus y Eubacterias así como el aumento de Bacteroides y Enterobacteria que contribuyen a la inflamación de bajo grado. Mantener determinado espectro de microbiota en el intestino es crítico para el estado saludable (Singh et al., 2017).

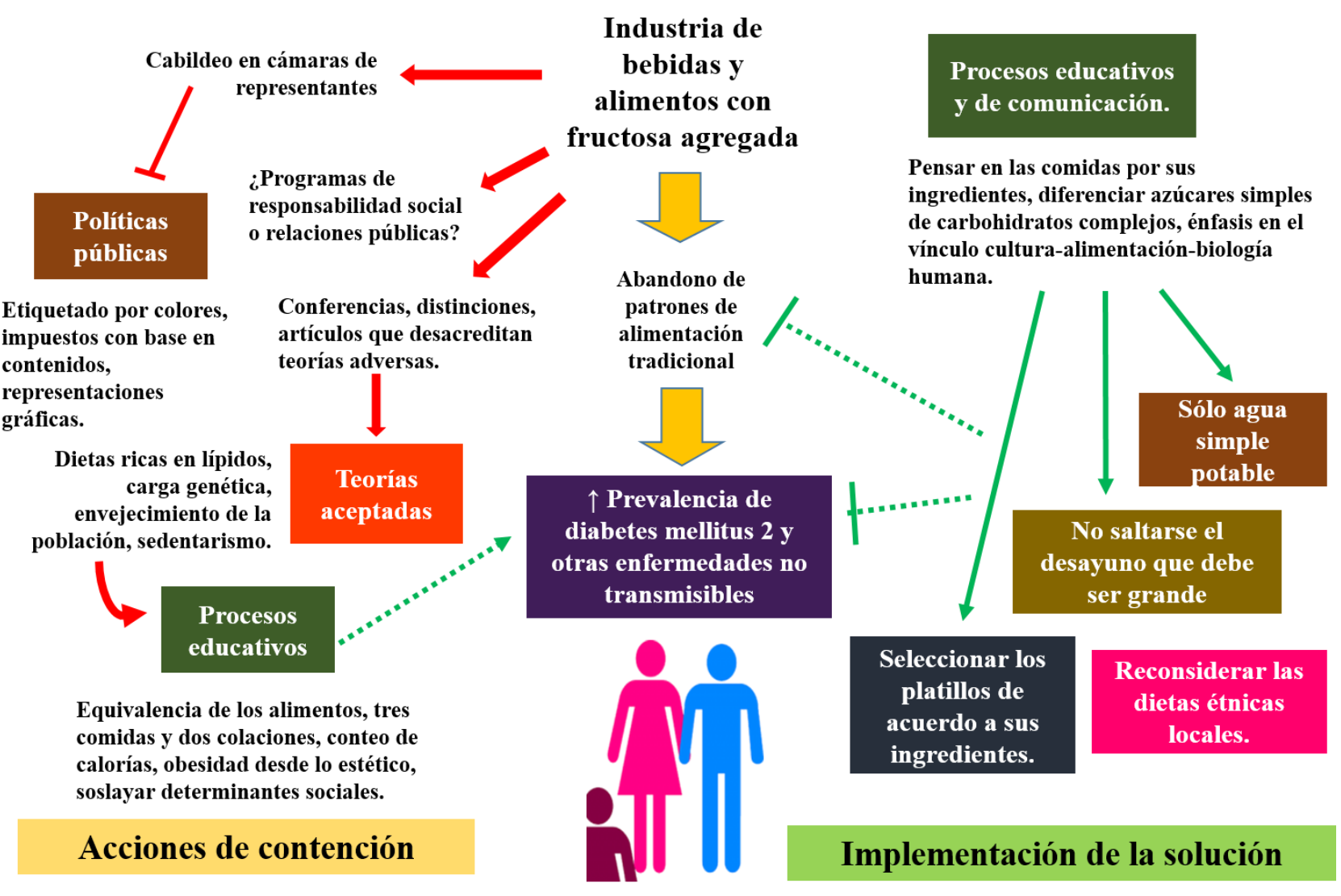

Fig. 1. Dinámica acerca de bebidas y alimentos de la industria.

Para las enfermedades monogénicas es relevante la secuencia de bases, para las poligénicas el sustrato de alelos de cada individuo lo hace susceptible determinadas condiciones del ambiente. El estrés sobre el gen produce ENT en la mayoría de las personas (Johnson et al., 2009). El efecto en el genoma del consumo de las dietas de tipo moderno es complejo pero cada vez se comprende mejor. Un efecto primordial del ambiente social, pero no el único, ocurre en la perturbación de la expresión de genes de miARN. Éstos regulan la expresión de los genes que codifican para proteínas. La expresión de genes de miARN aumenta bajo condiciones específicas como etapas del crecimiento, estrés y señales extracelulares. Una de estas señales extracelulares es el aumento de sacarosa por su contenido de fructosa consecuencia del consumo de bebidas y alimentos con azúcar visible u oculta (Sud et al., 2017; Su et al., 2017; Baena et al., 2016). .

\section{Implementación de la solución}

Ya que se trata de un problema complejo la solución no puede ser simple y debe ser analizada con base en información científica en los procesos educativos. Si la causa principal de las modificaciones metabólicas que se presentan como diabetes mellitus de tipo 2 es la dieta moderna, especialmente por el contenido de azúcar añadida, oculta o no, la solución primordial ha de ser la modificación en el estilo de vida (Figura 1). Esto comprende al menos tres aspectos. Evitar el azúcar en las bebidas. No saltarse el desayuno que debe ser grande y evitar la cena. Seleccionar los alimentos con base en los ingredientes de los platos y preferir los de la cocina tradicional. Esto infiere modificaciones en la formación de los profesionales de la salud para ofrecer esas soluciones a las personas bajo su cuidado.

Beber sólo agua simple potable. Las bebidas que se promueven para los niños, leches con sabor, néctares y jugos, tienen altas cantidades de azúcar. El consumo de bebidas azucaradas no produce sensación de saciedad (Teff et al., 2004). El metabolismo humano no tiene respuesta al azúcar diluido, al beberlo no se produce la fase cefálica que favorece la liberación de insulina, pues no se libera el polipéptido pancreático, por consiguiente, tampoco se produce la fase vagal de la homeostasis de la glucosa. No se recomiendan las bebidas con edulcorantes artificiales. Quienes toman estas bebidas, sobre todo niños y adolescentes, no distinguen claramente la diferencia entre endulzadas con azúcar o edulcorantes. Esto tiene como 
consecuencia que no contribuyen a disminuir la ingesta de calorías ya que con facilidad se beben refrescos con o sin azúcar de manera indistinta. Es posible que esta sea la causa de la asociación entre el consumo de bebidas con edulcorantes y obesidad así como DM2 (Shearer y Swithers, 2016; Yarmolinsky et al., 2016). Otra consecuencia son las modificaciones en la microbiota y a su vez modificaciones en la función del intestino grueso. Experimentos en animales han demostrado aumento de la concentración de colesterol y ácido cólico en hígado posteriormente a la ingesta de endulzantes como la sucralosa así como aumento de iNOS y TNF$\alpha$ en hígado que son mediadores de la inflamación, a consecuencia de la ingestión de sacarina (Uebanso et al., 2017; Bian et al., 2017). Otros estudios han demostrado que el consumo de sucralosa pueden aumentar la motilidad intestinal y agravan el intestino irritable (Spencer et al., 2016).

Tabla 2. Comparativo de los programas de estudios de medicina relacionados con la nutrición

\begin{tabular}{|c|c|c|c|c|}
\hline Universidad & $\begin{array}{l}\text { Asignaturas } \\
\text { relacionadas con salud } \\
\text { nutricional }\end{array}$ & $\begin{array}{l}\text { Semestre en el que } \\
\text { se imparten }\end{array}$ & $\begin{array}{l}\text { Total de horas e } \\
\text { impartidas por } \\
\text { semana }\end{array}$ & Área de formación \\
\hline \multirow{3}{*}{$\begin{array}{l}\text { Universidad } \\
\text { Nacional Autónoma } \\
\text { de México, plan } \\
\text { vigente } 2010\end{array}$} & $\begin{array}{l}\text { Bioquímica y biología } \\
\text { molecular }\end{array}$ & $\begin{array}{l}\text { Primer y segundo } \\
\text { semestre }\end{array}$ & $\begin{array}{l}102 / 132 \text { horas } \\
\text { totales, } 60 \% \\
\text { teóricas y } 40 \% \\
\text { prácticas. }\end{array}$ & Bases biomédicas \\
\hline & Endocrinología & Séptimo semestre & $\begin{array}{l}90 \text { horas totales, } \\
34 \% \text { teóricas, } 66 \% \\
\text { prácticas }\end{array}$ & \multirow[b]{2}{*}{ Disciplina clínica } \\
\hline & Nutrición humana & Séptimo semestre & $\begin{array}{l}16 \text { horas totales, } \\
50 \% \text { teóricas, } 50 \% \\
\text { prácticas }\end{array}$ & \\
\hline \multirow{2}{*}{$\begin{array}{l}\text { Universidad Juárez } \\
\text { Autónoma de } \\
\text { Tabasco, plan } \\
\text { vigente } 2010\end{array}$} & $\begin{array}{l}\text { Bioquímica y biología } \\
\text { molecular }\end{array}$ & Segundo semestre & $\begin{array}{l}80 \text { horas totales, } \\
60 \% \text { teóricas y } 40 \% \\
\text { prácticas. }\end{array}$ & \multirow{2}{*}{$\begin{array}{l}\text { Área sustantiva } \\
\text { profesional }\end{array}$} \\
\hline & $\begin{array}{l}\text { Endocrinología, } \\
\text { metabolismo y } \\
\text { nutrición }\end{array}$ & Octavo semestre & $\begin{array}{l}80 \text { horas totales, } \\
60 \% \text { teóricas y } 40 \% \\
\text { prácticas. }\end{array}$ & \\
\hline \multirow{2}{*}{$\begin{array}{l}\text { Universidad } \\
\text { Autónoma del } \\
\text { Estado de Hidalgo, } \\
\text { plan vigente } 2010\end{array}$} & Bioquímica & Segundo semestre & No especificado & Básica \\
\hline & Salud y nutrición & Cuarto semestre & No especificado & Clínica \\
\hline $\begin{array}{l}\text { Universidad de San } \\
\text { Marcos, Facultad } \\
\text { de San Fernando, } \\
\text { plan vigente } 2018\end{array}$ & Bioquímica & Cuarto semestre & No especificado & Estudios generales \\
\hline $\begin{array}{l}\text { Universidad de } \\
\text { Buenos Aires, } \\
\text { República } \\
\text { Argentina, plan } \\
\text { vigente } 2009\end{array}$ & Nutrición & $\begin{array}{l}\text { Séptimo y octavo } \\
\text { semestres }\end{array}$ & No especificado & Básica \\
\hline \multirow{4}{*}{$\begin{array}{l}\text { Universidad de } \\
\text { Sevilla, España, } \\
\text { plan vigente } 2015\end{array}$} & $\begin{array}{l}\text { Bioquímica y biología } \\
\text { molecular básica }\end{array}$ & Primer semestre & No especificado & Básica \\
\hline & $\begin{array}{l}\text { Bioquímica y biología } \\
\text { molecular humana }\end{array}$ & Primer semestre & No especificado & Básica \\
\hline & $\begin{array}{l}\text { Bioquímica clínica y } \\
\text { patología molecular }\end{array}$ & Quinto semestre & No especificado & Médica \\
\hline & $\begin{array}{l}\text { Patología médica del } \\
\text { sistema endócrino, } \\
\text { reumatología, } \\
\text { nefrología y sistema } \\
\text { nervioso }\end{array}$ & Quinto semestre & No especificado & Médica \\
\hline \multirow{2}{*}{$\begin{array}{l}\text { Universidad de } \\
\text { Madrid, España, } \\
\text { plan vigente }\end{array}$} & Bioquímica básica & Primer semestre & $\begin{array}{l}150 \text { horas totales } \\
\text { (cada crédito } \\
\text { equivale a } 25 \text { horas } \\
\text { de trabajo del } \\
\text { alumno); total de } \\
\text { créditos: } 6\end{array}$ & Básica \\
\hline & Bioquímica humana & Segundo semestre & $\begin{array}{l}150 \text { horas totales } \\
\text { (cada crédito } \\
\text { equivale a } 25 \text { horas } \\
\text { de trabajo del } \\
\text { alumno); total de } \\
\text { créditos: } 6\end{array}$ & Médica \\
\hline
\end{tabular}


No saltarse el desayuno que debe ser grande (y evitar la cena). La modificación del patrón de consumo de alimentos de diurno a vespertino y nocturno se contrapone al patrón de liberación circádica de cortisol y de la sensibilidad de la insulina (Chan y Debono, 2010; Carrasco et al., 2016). El principal efecto del cortisol es catabólico, y un mensajero entre los relojes internos central y periférico (Chan, 2016). Su liberación sigue una curva que inicia alrededor de las 4 de la mañana que se acelera hacia las 7, lo que se llama respuesta del cortisol al despertar (Fries et al, 2009), logra su acmé hacia las 9 de la mañana y tiene una caída prolongada hasta las 7 de la tarde. En este periodo se disminuye la síntesis de proteínas y el depósito de ellas, como la albúmina sérica. De día hay más aminoácidos en sangre que entran al ciclo de gluconeogénesis y en general se considera que tiende a elevar la glucemia porque disminuye la actividad de la insulina en tejidos muscular y adiposo. También se movilizan las reservas de ácidos grasos desde el tejido graso. Durante la noche, con mínimos niveles de cortisol, hay un periodo de anabolismo con reparación tisular, entre ellos el muscular. La curva determina la mejor hora para beber café, 11 de la mañana y 4 de la tarde cuando baja el cortisol (Nakamura et al., 2008), así como la hora para comer el pastel de chocolate (Jakubowicz et al., 2011).

Debido a la liberación circádica de hormonas como cortisol y de crecimiento hay diferencias en los procesos fisiológicos. De día se es más sensible al sabor dulce; ello disminuye hacia las horas finales del día. En quienes no desayunan aumenta la leptina pero se produce resistencia en los receptores centrales por lo cual deja de tener función como señal de saciedad (Asao et al., 2016). En niños que desayunan el desarrollo escolar es mejor y tienen menos factores de riesgo cardiovascular y de síndrome metabólico independientemente de la calidad de la dieta (Hoa et al., 2015), en mujeres que no desayunan es más probable la hipertensión arterial (Witbracht et al., 2015). En pacientes con diabetes un desayuno grande disminuyó la hemoglobina glicosilada y la necesidad de medicamentos (Rabinovitz et al., 2014); si contiene carbohidratos complejos promueve la sensación de saciedad. La sensibilidad a la insulina es diferente a diversas horas del día: mayor a medio día y menor a media noche lo que es modulado en un reloj circadiano del tejido adiposo independiente de los otros relojes (Jakubowicz et al., 2011). Saltarse el desayuno se relaciona fuertemente con aterosclerosis, sintomática o no (Uzhova et al., 2017).

Seleccionar los platos de acuerdo a los ingredientes (la comida en su contexto). Seleccionar comida saludable no es más caro que preferir comida industrializada moderna (Rao et al., 2013). La elaboración de las comidas, y no el énfasis en los nutrimentos, es lo relevante en nutrición (Jacobs y Tapsell, 2007). Las comidas, además, se deben considerar en un contexto biológico y cultural, no desde la época paleolítica sino en función de la selección producida por la introducción de la agricultura. Son conocidos los beneficios metabólicos de la cocina mediterránea (Leighton et al., 2009). Son semejantes los de la cocina tradicional japonesa (Inoue et al., 2014), libanesa (Naja et al., 2012), noruega (Bjørnarå et al., 2015), mexicana (Muñoz et al., 2017; Flores et al., 2010). También es efectiva como tratamiento de las personas con depresión (Jacka et al, 2017). Los alimentos elaborados según recetas tradicionales tienen más posibilidades de aportar, además de macro, micronutrimentos y variedad de oligoelementos, una mayor cantidad de probióticos, prebióticos, simbióticos, compuestos funcionales, así como fibras solubles e insolubles (Mercado, 2012).

Promover la actividad física. Es parte de la prevención y el manejo de la diabetes mellitus y los problemas cardiometabólicos. Aumenta la masa muscular y disminuye el porcentaje de grasa, mejora la capacidad aeróbica, disminuye la tensión arterial y eleva las lipoproteínas de alta densidad, aumenta la sensibilidad a la insulina y mejora el metabolismo de los triglicéridos (Howe et al, 2011; Schenck y Horowitz, 2007), aumenta la consistencia del hueso, mejora el estado de bienestar mediante la liberación de testosterona y de endorfinas, tiene efectos antidepresivos (Harvey et al, 2017; Ernst et al., 2006), propicia la neurogenesis y el aprendizaje (Sylvia et al., 2010; Van Prag et al., 2005; Cotman y Berchold, 2002), disminuye la dependencia y las caídas en ancianos, disminuye la probabilidad de desarrollar cáncer de colon, mama y próstata (Nickels et al., 2013).

Construir las capacidades necesarias en el personal de salud. Ya sea por escaso tiempo para atender a los pacientes, pocas habilidades del personal de salud, escaso material de apoyo o prejuicios acerca de los pacientes, éstos no reciben indicaciones efectivas (Dacey et al, 2012; Ball et al, 2010; Wynn et al, 2010). Otra barrera, quizás la más importante, es la escasa formación que se logra durante los procesos de educación médica (Salen et al, 2015). En Estados Unidos sólo 27\% de las escuelas de medicina cubren el mínimo de 25 horas de un curso acerca de alimentos, que es una recomendación de la Academia Nacional de Ciencias de ese país (Eng, 2015). Evaluaciones con pobres resultados acerca de nutrición por parte de estudiantes y egresados de medicina datan de 1991. A consecuencia, la Fundación Mexicana para la Salud (FUNSANUT) y la Asociación Nacional de Escuelas y Facultades de Medicina (ANFEN) de México coincidieron en la necesidad de fortalecer la educación en nutrición desde el pregrado (Casanueva y Valdéz, 1991). Los cursos que se originaron a partir de esas observaciones fueron de distintos enfoques. También se crearon en la lógica de que, "para aprender cabalmente la nutrición" (Vega e Iñárritu, 2001), es necesario aprender primero bioquímica, biología molecular y fisiología. Esa forma de construir el curriculum no considera los avances de las neurociencias, las que si se toman en cuenta desde la mercadotecnia (Tabla 2). 


\section{DISCUSION}

Por ser una revisión integrativa es un análisis amplio de un campo de estudio que tiene muchas aristas. Se hace la identificación de una raíz principal que debe ser el objeto de los procedimientos de prevención de la enfermedad y de sus comorbilidades, como el eje de la terapia no farmacológica. Aunque se encontraron revisiones donde se minimiza el efecto de la fructosa en las modificaciones metabólicas, fenotípicas y epigenéticas, sus autores tenían conflicto de interés y recibieron financiamiento de la industria del azúcar. La evidencia muestra, por el contrario, la vía cerrada a la que conduce la presencia de fructosa o sacarosa agregada a alimentos y bebidas.

En otro sentido, los enfoques desde los que parten las medidas de contención no son todo lo fuertes que deberían con base en los datos que surgen de la investigación científica. Algunas de ellas sólo son creencias y no tienen confirmación por lo que eventualmente producen lo que pretender evitar. Es necesario construir recomendaciones que se basen en la biología humana y la interacción biología-ambiente. Entre otras, esta conexión hace dependiente a las personas de las dietas tradicionales pues en el proceso evolutivo hubo selección de los individuos que podían nutrirse sin complicaciones con los productos del entorno. Esto también tiene implicaciones en la estrecha interacción entre la microbiota intestinal y la salud.

En este caso, para transitar desde patrones de consumo escasamente saludables hacia capacidades para la promoción de la alimentación saludable es necesaria la integración de cocina, ciencia, medicina y nutrición. Es también necesario considerar patrones históricos de alimentación (Berciano y Ordovás, 2014) tales como las cocinas étnicas locales pues hay un innegable vínculo entre los genes y los productos locales con que se han preparado las comidas tradicionales (Román et al, 2013) ya que el invento de la agricultura data de al menos 10 mil años en las distintas regiones del mundo, lo que produjo procesos de selección natural.

La integración de capacidades para proporcionar consejos prácticos hacia el estilo de vida saludable requiere de transformaciones en la educación en nutrición, incluso en forma de talleres donde se enseñe a cocinar alimentos saludables (Eisenberg y Burgess, 2015). El aprendizaje de la preparación de los alimentos por parte del personal de salud en clases de nutrición "para médicos", aunque no exclusivamente para ellos, tiene la finalidad de sentar las bases de considerar que lo que se come tiene un proceso de elaboración que no sólo resulta en textura y sabor sino en el concepto de "pensar en las comidas de manera integral".

\section{CONCLUSIONES}

De acuerdo al trabajo presentado y a los resultados obtenidos, se pueden plantear las siguientes conclusiones principales:

1.- La tendencia en la prevalencia de DM2 y ENT se encuentra en incremento a pesar de los esfuerzos de los sistemas de salud. 2. Las acciones de contención como prescripción de actividad física, medicamentos, etiquetado a alimentos y bebidas, restricción de venta en espacios educativos y de salud, impuestos a bebidas con azúcar o edulcorantes artificiales no han sido efectivas en su propósito. 3. Estas acciones se contrarrestan por la falta de regulación a la industria de bebidas y alimento. 4. Si bien los genes y el envejecimiento de la población son influyentes, la adición de fructosa a bebidas y alimentos es determinante en la pandemia de DM2 y ENT. 5. Para que los profesionales de la salud apliquen los lineamientos de las guías clínicas para prevención y tratamiento de DM2 y ENT es necesario modificar la curricula de las escuelas de medicina. 6 . Los procesos de formación de profesionales de la salud deben incorporar conceptos sobre la comida étnica tradicional.

\section{REFERENCIAS}

Alemán, J.A., de Baranda, P. S., y otro autor, Guía para la Prescripción de Ejercicio Físico en Pacientes con Riesgo Cardiovascular, Seh-Lelha. (2014).

Alberti, G., Zimmet, P., y otros cuatro autores, Type 2 diabetes in the young: the evolving epidemic: the international diabetes federation consensus workshop, 10.2337/diacare.27.7.1798, Diabetes Care, 27(7), 1798-1811 (2004).

American Diabetes Association (ADA), So... Do I have prediabetes?

https://doihaveprediabetes.org/?loc=dorg_201707_en_feat_adcouncil (2019).

Aroda, V. R., Knowler, W.C., y otros cinco autores, Metformin for diabetes prevention: Insights gained from the diabetes prevention program/diabetes prevention program outcomes study, 10.1007/s00125-017-4361-9. Diabetologia, 60(9), 1601-1611 (2017).

Asao, K., Marekani, A.S., y otros dos autores, Leptin level and skipping breakfast: The national health and nutrition examination survey III (NHANES III), 10.3390/nu8030115. Nutrients, 8(3), 115 (2016). 
Baena, M., Sangüesa, G., y otros cinco autores, Fructose, but not glucose, impairs insulin signaling in the three major insulin-sensitive tissues, 10.1038/srep26149. Scientific Reports, 6, 26149, (2016).

Ball, L. E., Hughes, R.M., y otro autor, Nutrition in general practice: role and workforce preparation expectations of medical educators, https://doi.org/10.1071/PY10014. Australian Journal of Primary Health, 16(4), 304-310, 2010.

Barzilai, N., Crandall, J.P., y otros dos autores, Metformin as a tool to target aging, https://doi.org/10.1016/j.cmet.2016.05.011. Cell Metabolism, 23(6), 1060-1065, (2016).

Basaranoglu, M., Basaranoglu, G., y otros dos autores, Fructose as a key player in the development of fatty liver disease. 10.3748/wjg.v19.i8.1166. World Journal of Gastroenterology, 19(8), 1166 (2013).

Berciano, S., y Ordovás, J.M., Nutrition and cardiovascular health. https://doi.org/10.1016/j.rec.2014.05.003. Revista Española de Cardiología (Engl Ed), 67(9), 738-747 (2014).

Bian, X., Tu, P., y otros cuatro autores, Saccharin induced liver inflammation in mice by altering the gut microbiota and its metabolic functions. https://doi.org/10.1016/j.fct.2017.04.045. Food and Chemical Toxicology, 107, 530-539 (2017).

Bjørnarå, H. B., Hillesund, E.R., y otros cuatro autores, An assessment of the test-retest reliability of the New Nordic Diet score. https://doi.org/10.3402/fnr.v59.28397. Food \& Nutrition Research, 59(1), 28397 (2015).

Bray, G.A., Energy and fructose from beverages sweetened with sugar or high-fructose corn syrup pose a health risk for some people, doi: https://doi.org/10.3945/an.112.002816. Adv Nutrition, 4(2), 220-225 (2013)

Carrasco, M.P., Rivero, B., y otros ocho autores, Human adipose tissue expresses intrinsic circadian rhythm in insulin sensitivity. https://doi.org/10.1096/fj.201600269RR. The FASEB Journal, 30(9), 3117-3123 (2016).

Casanueva, E., y Valdéz, R., El conocimiento nutriológico de médicos residentes, Revista de Investigación Clínica 43,211214 (1991).

Chan, S., y Debono, M., Replication of cortisol circadian rhythm: new advances in hydrocortisone replacement therapy. https://doi.org/10.1177/2042018810380214. Therapeutic Advances in Endocrinology and Metabolism, 1(3), $129-138$. (2010).

Cobiac, L.J., Tam, K., y otros dos autores, Taxes and subsidies for improving diet and population health in Australia: a cost-effectiveness modelling study, doi: https://doi.org/10.1371/journal.pmed.1002232. PLoS Medicine, 14(2), e1002232 (2017).

Colberg, S. R., Sigal, R.J., y otros cinco autores, Exercise and type 2 diabetes: the American College of Sports Medicine and the American Diabetes Association: joint position statement, https://doi.org/10.2337/dc10-9990. Diabetes Care, 33(12), e147-e167 (2010).

Cotman, C.W., y Berchtold, N.C., Exercise: a behavioral intervention to enhance brain health and plasticity, https://doi.org/10.1016/S0166-2236(02)02143-4. TRENDS Neuroscience, 25(6), 295-301 (2002).

Dacey, M., Arnstein, F., y otros tres autores, The impact of lifestyle medicine continuing education on provider knowledge, attitudes, and counseling behaviors, https://doi.org/10.3109/0142159X.2012.733459. Medical Teacher, 35(5), e1149e1156 (2013).

Daly, M. E., Vale, C., y otros tres autores, Dietary carbohydrates and insulin sensitivity: a review of the evidence and clinical implications, https://doi.org/10.1093/ajcn/66.5.1072. The American Journal of Clinical Nutrition, 66(5), 1072-1085 (1997).

DeFronzo, R.A., From the triumvirate to the ominous octet: a new paradigm for the treatment of type 2 diabetes mellitus. Diabetes, 58(4),773-795 (2009).

Dehghan, A., Van Hoek, M., y otros tres autores, High serum uric acid as a novel risk factor for type 2 diabetes, doi: https://doi.org/10.2337/dc07-1276. Diabetes Care, 31(2), 361-362 (2008).

DiNicolantonio, J.J., O'keefe, J.H., y otro autor, Added fructose: a principal driver of type 2 diabetes mellitus and its consequences, https://doi.org/10.1016/j.mayocp.2014.12.019. Mayo Clinic Proceedings, 90(3), 372-381 (2015)

Douard, V., y Ferraris, R.P., Regulation of the fructose transporter GLUT5 in health and disease, https://doi.org/10.1152/ajpendo.90245.2008. American Journal of Physiology-Endocrinology and Metabolism, 295(2), E227-E237 (2008).

Martínez, Á., Montaner, G. I., y otro autor, Lifestyle, dietetic habits and overweight and obesity prevalence in a pediatric population. Revista Pediatría Atención Primaria, 12(45), 53-65 (2010).

Eisenberg, D.M., y Burgess, J.D., Nutrition education in an era of global obesity and diabetes: thinking outside the box, doi: 10.1097/ACM.0000000000000682. Academic Medicine, 90(7), 854-860.

Elliott, S.S., Keim, N.L., y otros tres autores, Fructose, weight gain, and the insulin resistance syndrome, https://doi.org/10.1093/ajcn/76.5.911. The American Journal of Clinical Nutrition, 76(5), 911-922 (2002).

Ernst, C., Olson, A.K., y otros tres autores, Antidepressant effects of exercise: evidence for an adult-neurogenesis hypothesis? Journal of Psychiatry \& Neuroscience. 31(2), 84-92, (2006).

Flegal, K.M., Kruszon, D., y otros tres autores, Trends in obesity among adults in the United States, 2005 to 2014, doi:10.1001/jama.2016.6458. JAMA, 315(21), 2284-2291, (2016). 
Flores, M., Macias, N., y otros cinco autores, Dietary Patterns in Mexican Adults Are Associated with Risk of Being Overweight or Obese, https://doi.org/10.3945/jn.110.121533. The Journal of Nutrition, 140(10), 1869-1873 (2010).

Fries, E., Dettenborn, L., y otro autor, The cortisol awakening response (CAR): facts and future directions, https://doi.org/10.1016/j.jpsycho.2008.03.014. International Journal of Psychophysiology, 72(1), 67-73 (2009).

Gaby, A.R., Adverse effects of dietary fructose, Alternative Medicine Review, 10(4), 294, (2005).

Gómez, H., Fullman, N., y otros cinco autores, Dissonant health transition in the states of Mexico, 1990-2013: a systematic analysis for the Global Burden of Disease Study 2013, https://doi.org/10.1016/S0140-6736(16)31773-1. The Lancet, 388(10058), 2386-2402 (2016).

Goran, M.I., Dumke, K., y otros cuatro autores, The obesogenic effect of high fructose exposure during early development, https://doi.org/10.1038/nrendo.2013.108. Nature Reviews Endocrinology, 9(8), 494 (2013).

Harvey, S. B., Øverland, S., y otros cuatro autores, Exercise and the prevention of depression: results of the HUNT Cohort Study, https://doi.org/10.1176/appi.ajp.2017.16111223. American Journal of Psychiatry, 175(1), 28-36 (2017).

Havel, P.J., Dietary fructose: implications for dysregulation of energy homeostasis and lipid/carbohydrate metabolism, https://doi.org/10.1111/j.1753-4887.2005.tb00132.x. Nutrition Reviews, 63(5), 133-157 (2005).

Ho, C. Y., Huang, Y.C., y otros tres autores, Breakfast is associated with the metabolic syndrome and school performance among Taiwanese children, https://doi.org/10.1016/j.ridd.2015.07.003. Research in Developmental Disabilities, 43, 179188 (2015).

Howe, C.A., Harris, R.A., y otro autor, 10-month physical activity intervention improves body composition in young black boys, doi:10.1155/2011/358581. Journal of Obesity (2011).

Inoue, H., Sasaki, R., y otros dos autores, Short-term intake of a Japanese-style healthy lunch menu contributes to prevention and/or improvement in metabolic syndrome among middle-aged men: a non-randomized controlled trial, https://doi.org/10.1186/1476-511X-13-57. Lipids in Health and Disease, 13(1), 57 (2014).

Instituto Nacional de Salud Pública (INSP), Encuesta Nacional de Salud y Nutrición de Medio Camino, 2016, Secretaría de Salud de México (2016).

Jacka, F. N., O'Neil, A., y otros cinco autores, A randomised controlled trial of dietary improvement for adults with major depression (the 'SMILES'trial), https://doi.org/10.1186/s12916-017-0791-y. BMC medicine, 15(1), 23 (2017).

Jacobs, D.R., y Tapsell, L.C., Food, not nutrients, is the fundamental unit in nutrition, https://doi.org/10.1111/j.17534887.2007.tb00269.x. Nutrition Reviews, 65(10), 439-450 (2007).

Jakubowicz, D., Froy, O., y otros dos autores, Meal timing and composition influence ghrelin levels, appetite scores and weight loss maintenance in overweight and obese adults, https://doi.org/10.1016/j.steroids.2011.12.006. Steroids, 77(4), 323-331 (2012).

Johnson, R.J., Segal, M.S., y otros cinco autores, Potential role of sugar (fructose) in the epidemic of hypertension, obesity and the metabolic syndrome, diabetes, kidney disease, and cardiovascular disease, https://doi.org/10.1093/ajcn/86.4.899. The American Journal of Clinical Nutrition, 86(4), 899-906 (2007).

Johnson, R.J., Perez, S.E., y otros cinco autores, Hypothesis: could excessive fructose intake and uric acid cause type 2 diabetes?, https://doi.org/10.1210/er.2008-0033. Endocrine Reviews, 30(1), 96-116 (2009).

Johnson, R.J., Nakagawa, T., y otros siete autores, Sugar, uric acid, and the etiology of diabetes and obesity, https://doi.org/10.2337/db12-1814. Diabetes, 62(10), 3307-3315 (2013).

Kahn, B.B., y Flier, J.S., Obesity and insulin resistance, https://doi.org/10.1172/JCl10842. The Journal of Clinical Investigation, 106(4), 473-481 (2000).

Kaplík, P., Prístavka, M., y otros dos autores, Use of $8 D$ method to solve problems, https://doi.org/10.4028/www.scientific.net/AMR.801.95. Advanced Materials Research, (801), 95-101 (2013).

Kearns, C.E., Schmidt, L.A., y otro autor, Sugar industry and coronary heart disease research: a historical analysis of internal industry documents, doi:10.1001/jamainternmed.2016.5394. JAMA Internal Medicine, 176(11), 1680-1685 (2016).

King, H., Aubert, R.E., y otro autor, Global burden of diabetes, 1995-2025: prevalence, numerical estimates, and projections, doi https://doi.org/10.2337/diacare.21.9.1414. Diabetes Care, 21(9), 1414-1431 (1998).

Kohen, A. R., Theriault, A., y otro autor, Emergence of the metabolic syndrome in childhood: an epidemiological overview and mechanistic link to dyslipidemia, doi: https://doi.org/10.1016/S0009-9120(03)00038-9. Clinical Biochemistry, 36(6), 413-420 (2003).

Laville, M., y Nazare, J.A., Diabetes, insulin resistance and sugars, https://doi.org/10.1111/j.1467-789X.2008.00562.x. Obesity Reviews, 10, 24-33 (2009).

Leighton, F., Polic, G., y otros diez autores, Health impact of Mediterranean diets in food at work, https://doi.org/10.1017/S1368980009990486. Public Health Nutrition, 12(9A), 1635-1643 (2009).

Lim, J.S., Mietus, S.M., y otros tres autores, The role of fructose in the pathogenesis of NAFLD and the metabolic syndrome, https://doi.org/10.1038/nrgastro.2010.41. Nature Reviews Gastroenterology and Hepatology, 7(5), 251 (2010). 
Lin, H.V., Frassetto, A., y otros cinco autores, Butyrate and propionate protect against diet-induced obesity and regulate gut hormones via free fatty acid receptor 3-independent mechanisms, 10.1371/journal.pone.0035240. PloS One, 7(4), e35240 (2012).

Loomba, R., y Sanyal, A.J., The global NAFLD epidemic, https://doi.org/10.1038/nrgastro.2013.171. Nature Reviews Gastroenterology \& Hepatology, 10(11), 686 (2013).

MacDonald, I.A., A review of recent evidence relating to sugars, insulin resistance and diabetes, https://doi.org/10.1007/s00394-016-1340-8. European Journal of Nutrition, 55(2), 17-23 (2016).

Metcalf, B. S., Hosking, J., y otros cuatro autores, Fatness leads to inactivity, but inactivity does not lead to fatness: a longitudinal study in children (EarlyBird 45), http://dx.doi.org/10.1136/adc.2009.175927. Archives of Disease in Childhood, 96(10), 942-947 (2011).

Moreira, P. I., High-sugar diets, type 2 diabetes and Alzheimer's disease, 10.1097/MCO.0b013e328361c7d1. Current Opinion in Clinical Nutrition \& Metabolic Care, 16(4), 440-445 (2013).

Mozaffarian, D., Foods, obesity, and diabetes - are all calories created equal?, https://doi.org/10.1093/nutrit/nuw024. Nutrition Reviews, 75(suppl_1), 19-31 (2017).

Mozaffarian, D., Conflict of interest and the role of the food industry in nutrition research, doi:10.1001/jama.2017.3456. JAMA, 317(17), 1755-1756 (2017).

Muñoz, J.M., Cordova, J.A., y otros dos autores, Effect of substituting usual lunch modernized meal with traditional based meal on biochemical parameters in young women. Wulfenia Journal., 24, 357-370 (2017).

Naja, F., Hwalla, N., y otros cinco autores, Dietary patterns and odds of Type 2 diabetes in Beirut, Lebanon: a case-control study, doi: https://doi.org/10.1186/1743-7075-9-111. Nutrition \& Metabolism, 9(1), 111 (2012).

Nakamura, Y., Sanematsu, K., y otros seis autores, Diurnal variation of human sweet taste recognition thresholds is correlated with plasma leptin levels, https://doi.org/10.2337/db07-1103. Diabetes, 57(10), 2661-2665 (2008).

Nickels, S., Truong, T., y otros cinco autores, Evidence of gene-environment interactions between common breast cancer susceptibility loci and established environmental risk factors, https://doi.org/10.1371/journal.pgen.1003284. PLoS Genetics, 9(3), e1003284 (2013).

Nomura, K., y Yamanouchi, T., The role of fructose-enriched diets in mechanisms of nonalcoholic fatty liver disease, https://doi.org/10.1016/j.jnutbio.2011.09.006. The Journal of Nutritional Biochemistry, 23(3), 203-208 (2012).

Out, M., Miedema, I., y otros cinco autores, Metformin-associated prevention of weight gain in insulin-treated type 2 diabetic patients cannot be explained by decreased energy intake: A post hoc analysis of a randomized placebo-controlled 4.3year trial. Diabetes, https://doi.org/10.1111/dom.13054. Obesity and Metabolism, 20(1), 219-223 (2018).

Pan American Health Organization (PAHO). Health in the Americas, 2017 Edition. Summary: Regional Outlook and Country Profiles. Washington, D.C. (2017).

Peña, M., y Bacallao, J., Obesity and Poverty: A New Public Health Challenge. Scientific Publication 576. D. C.: Organización Panamericana de la Salud (2000).

Panasevich, M.R., Peppler, W.T., y otros tres autores, Microbiome and NAFLD: potential influence of aerobic fitness and lifestyle modification, https://doi.org/10.1152/physiolgenomics.00012.2017. Physiological Genomics, 49(8), 385-399 (2017).

Rabinovitz, H.R., Boaz, M., y otros cinco autores, Big breakfast rich in protein and fat improves glycemic control in type 2 diabetics, https://doi.org/10.1002/oby.20654. Obesity, 22(5), E46-E54 (2014).

Rao, M., Afshin, A., y otros dos autores, Do healthier foods and diet patterns cost more than less healthy options? A systematic review and meta-analysis, 10.1136/bmjopen-2013-004277. BMJ Open, 3(12), e004277 (2013).

Rey, J.P., y Gonzalez, C.A., Research partnerships between Coca-Cola and health organizations in Spain. Eur J Public Health, 10.1093/eurpub/cky175 (2018).

Rho, Y. H., Zhu, Y., y otro autor, The epidemiology of uric acid and fructose, https://doi.org/10.1016/j.semnephrol.2011.08.004. Seminars in Nephrology, 31(5), 410-419 (2011).

Richelsen, B., Sugar-sweetened beverages and cardio-metabolic disease risks, 10.1097/MC0.0b013e328361c53e. Current Opinion in Clinical Nutrition \& Metabolic Care, 16(4), 478-484 (2013).

Rivera, J.A., Muñoz, O., y otros cuatro autores, Beverage consumption for a healthy life: recommendations for the Mexican population. Boletín Médico del Hospital Infantil de México, 65(3), 208-237 (2008).

Rodrigo, S., Rodríguez, L., y otros cinco autores, Fructose during pregnancy provokes fetal oxidative stress: The key role of the placental heme oxygenase-1, https://doi.org/10.1002/mnfr.201600193. Molecular Nutrition \& Food Research, 60(12), 2700-2711 (2016).

Román, S., Ojeda, C., y otro autor, Genética y evolución de la alimentación de la población en México. Revista de Endocrinología y Nutrición 21, 42-51 (2013).

Rorsman, P., Eliasson, L., y otros cuatro autores, The cell physiology of biphasic insulin secretion, https://doi.org/10.1152/physiologyonline.2000.15.2.72. Physiology, 15(2), $72-77$ (2000). 
Ruiz, B., Dijck, D.J., y otro autor, The relation of saturated fatty acids with low-grade inflammation and cardiovascular disease, https://doi.org/10.1016/j.jnutbio.2015.12.007. The Journal of Nutritional Biochemistry, 36, 1-20 (2016).

Samuel, V.T., Fructose induced lipogenesis: from sugar to fat to insulin resistance, https://doi.org/10.1016/j.tem.2010.10.003. Trends in Endocrinology \& Metabolism, 22(2), 60-65 (2011).

Sayiner, M., Koenig, A., y otros dos autores, Epidemiology of nonalcoholic fatty liver disease and nonalcoholic steatohepatitis in the United States and the rest of the world, https://doi.org/10.1016/j.cld.2015.10.001. Clinics in Liver Disease, 20(2), 205-214 (2016).

Schenk, S., y Horowitz, J.F., Acute exercise increases triglyceride synthesis in skeletal muscle and prevents fatty acidinduced insulin resistance, https://doi.org/10.1172/JCl30566. The Journal of clinical investigation, 117(6), 1690-1698 (2007).

Segal, M.S., Gollub, E., y otro autor, Is the fructose index more relevant with regards to cardiovascular disease than the glycemic index?, https://doi.org/10.1007/s00394-007-0680-9. European Journal of Nutrition, 46(7), 406 (2007).

Shearer, J., y Swithers, S.E., Artificial sweeteners and metabolic dysregulation: lessons learned from agriculture and the laboratory, https://doi.org/10.1007/s11154-016-9372-1. Reviews in Endocrine and Metabolic Disorders, 17(2), 179-186 (2016).

Singh, R.K., Chang, H.W., y otros cinco autores, Influence of diet on the gut microbiome and implications for human health, https://doi.org/10.1186/s12967-017-1175-y. Journal of Translational Medicine, 15(1), 73 (2017).

Slyper, A.H., The influence of carbohydrate quality on cardiovascular disease, the metabolic syndrome, type 2 diabetes, and obesity-an overview, https://doi.org/10.1515/jpem-2012-0419. Journal of Pediatric Endocrinology and Metabolism, 26(7-8), 617-629 (2013).

Spencer, M., Gupta, A., y otros cuatro autores, Artificial sweeteners: a systematic review and primer for gastroenterologists, 10.5056/jnm15206. Journal of Neurogastroenterology and Motility, 22(2), 168 (2016).

Spranger, J., Kroke, A., y otros cinco autores, Inflammatory cytokines and the risk to develop type 2 diabetes: results of the prospective population-based European Prospective Investigation into Cancer and Nutrition (EPIC)-Potsdam Study, https://doi.org/10.2337/diabetes.52.3.812. Diabetes, 52(3), 812-817 (2003).

Stanhope, K.L., Schwarz, J.M., y otro autor, Adverse metabolic effects of dietary fructose: results from recent epidemiological, clinical, and mechanistic studies, 10.1097/MOL.0b013e3283613bca. Current Opinion in Lipidology, 24(3), 198 (2013).

Su, Q., Sud, N., y otro autor, MicroRNAs in the pathogenesis of lipogenic diet induced hyperlipidemia and insulin resistance, The FASEB Journal, 31(1_s), Abstract Number: 299.8 (2017).

Sud, N., Zhang, H., y otros cuatro autores, Aberrant expression of microRNA induced by high-fructose diet: implications in the pathogenesis of hyperlipidemia and hepatic insulin resistance, https://doi.org/10.1016/j.jnutbio.2017.02.003. The Journal of Nutritional Biochemistry, 43, 125-131 (2017).

Sylvia, L.G., Ametrano, R.M., y otro autor, Exercise in the treatment of bipolar disorder: potential mechanisms of action mediated increase in neurogenesis and descent of the load allostatic, https://doi.org/10.1159/000270916. RET, 60, 27-35 (2010).

Tabung, F.K., Smith, S.A., y otros cinco autores, Development and validation of an Empirical Dietary Inflammatory Index, https://doi.org/10.3945/jn.115.228718. The Journal of Nutrition, 146(8), 1560-1570. DOI https://doi.org/10.3945/jn.115.228718 (2016).

Tappy, L., y Lê, K.A., Metabolic effects of fructose and the worldwide increase in obesity, https://doi.org/10.1152/physrev.00019.2009. Physiological Reviews, 90(1), 23-46 (2010).

Teff, K. L., Elliott, S.S., y otros cinco autores, Dietary fructose reduces circulating insulin and leptin, attenuates postprandial suppression of ghrelin, and increases triglycerides in women, https://doi.org/10.1210/jc.2003-031855. The Journal of Clinical Endocrinology \& Metabolism, 89(6), 2963-2972 (2004).

Ter, K.W., Schene, M.R., y otros tres autores, Effect of fructose consumption on insulin sensitivity in nondiabetic subjects: a systematic review and meta-analysis of diet-intervention trials, https://doi.org/10.3945/ajcn.116.137786. The American Journal of Clinical Nutrition, 104(6), 1562-1576 (2016).

Tseng, E., Yeh, H.C., y otro autor, Metformin use in prediabetes among US adults, 2005-2012, https://doi.org/10.2337/dc16-1509. Diabetes Care, dc161509 (2017).

Uebanso, T., Ohnishi, A., y otros seis autores, Effects of low-dose non-caloric sweetener consumption on gut microbiota in mice, https://doi.org/10.3390/nu9060560. Nutrients, 9(6), 560 (2017).

Uzhova, I., Fuster, V., y otros nueve autores, The importance of breakfast in atherosclerosis disease: insights from the PESA study, 10.1016/j.jacc.2017.08.027. Journal of the American College of Cardiology, 70(15), 1833-1842 (2017).

Van, H., Shubert, T., y otros dos autores, Exercise enhances learning and hippocampal neurogenesis in aged mice, https://doi.org/10.1523/JNEUROSCI.1731-05.2005. Journal of Neuroscience, 25(38), 8680-8685 (2005).

Vega, L., y Iñárritu, M.C., La enseñanza de la nutrición en la carrera de medicina. Revista de la Facultad de Medicina de la UNAM, 44(5), 224-229 (2001). 
Wei, Y., Wang, D., y otros dos autores, Fructose-mediated stress signaling in the liver: implications for hepatic insulin resistance, https://doi.org/10.1016/j.jnutbio.2006.03.013. The Journal of Nutritional Biochemistry, 18(1), 1-9 (2007).

Whittemore, R., y Knafl, K., The integrative review: updated methodology, https://doi.org/10.1111/j.13652648.2005.03621.x. Journal of Advanced Nursing, 52(5), 546-553 (2005).

Willett, W.C., y Ludwig, D.S., The 2010 dietary guidelines—the best recipe for health? New England Journal of Medicine, 365(17), 1563-1565 (2011).

Witbracht, M., Keim, N.L., y otros tres autores, Female breakfast skippers display a disrupted cortisol rhythm and elevated blood pressure, https://doi.org/10.1016/j.physbeh.2014.12.044. Physiology \& Behavior, 140, 215-221 (2015).

World Health Organization (WHO), Global report on diabetes. Geneva, Switzerland: World Health Organization; 86 pp. (2016).

Wynn, K., Trudeau, J.D., y otros dos autores, Nutrition in primary care: current practices, attitudes, and barriers. Canadian Family Physician, 56(3), e109-e116 (2010).

Yarmolinsky, J., Duncan, B.B., y otros cinco autores, Artificially sweetened beverage consumption is positively associated with newly diagnosed diabetes in normal-weight but not in overweight or obese Brazilian adults, https://doi.org/10.3945/jn.115.220194. The Journal of Nutrition, 146(2), 290-297 (2015).

Zimmet, P., Alberti, K.G., y otros dos autores, Diabetes mellitus statistics on prevalence and mortality: facts and fallacies, https://doi.org/10.1038/nrendo.2016.105. Nature Reviews Endocrinology, 12(10), 616 (2016). 